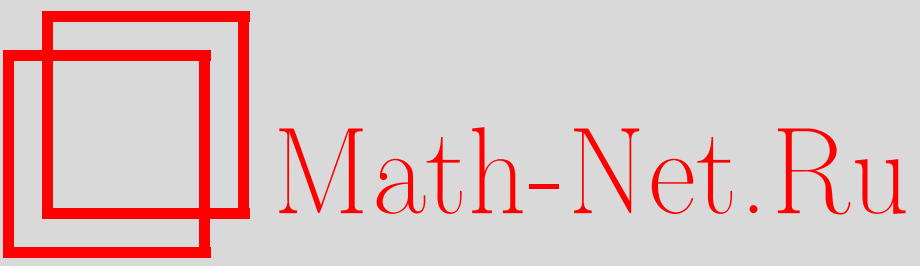

М. Ш. Бурлуцкая, А. В. Киселева, Я. П. Коржова, Классическое решение смешанной задачи для волнового уравнения на графе из двух ребер с циклом, Итоги науки и техн. Сер. Соврем. мат. и ее прил. Темат. обз., 2021, том 194, 78-91

DOI: https://doi.org/10.36535/0233-6723-2021-194-78-91

Использование Общероссийского математического портала Math-Net.Ru подразумевает, что вы прочитали и согласны с пользовательским соглашением

http://www.mathnet.ru/rus/agreement

Параметры загрузки:

IP : 54.198 .187 .58

26 апреля 2023 г., $16: 43: 13$ 


\title{
КЛАССИЧЕСКОЕ РЕШЕНИЕ СМЕШАННОЙ ЗАДАЧИ ДЛЯ ВОЛНОВОГО УРАВНЕНИЯ НА ГРАФЕ ИЗ ДВУХ РЕБЕР С ЦИКЛОМ
}

\author{
(C) 2021 г. \\ М. Ш. БУРЛУЦКАЯ, А. В. КИСЕЛЕВА, Я. П. КОРЖОВА
}

\begin{abstract}
АннотАция. В работе методом Фурье получено классическое решение смешанной задачи для волнового уравнения на простейшем геометрическом графе, состоящем из двух ребер, одно из которых образует цикл. Используется подход, базирующийся на методе контурного интегрирования резольвенты оператора, который позволяет с помощью специального преобразования формального ряда получить классическое решение задачи при минимальных условиях на начальные данные и при этом избежать трудоемкого исследования уточненных асимптотик собственных значений и собственных функций соответствующего оператора. Исследованы случаи непрерывного и суммируемого потенциалов.
\end{abstract}

Ключевъе слова: смешанная задача, волновое уравнение, граф, суммируемый потенциал, метод Фурье.

\section{CLASSICAL SOLUTION \\ OF THE MIXED PROBLEM FOR THE WAVE EQUATION \\ ON A GRAPH WITH TWO EDGES AND A CYCLE}

\author{
(c) 2021 M. SH. BURLUTSKAYA, A. V. KISELEVA, YA. P. KORZHOVA
}

\begin{abstract}
In this paper, using the Fourier method, we obtain a classical solution of the mixed problem for the wave equation on the simplest geometric graph consisting of two edges, one of which forms a cycle. We apply an approach based on the method of contour integration of the resolvent of an operator, which allows one to obtain a classical solution to the problem under minimal conditions on the initial data and, at the same time, to avoid a laborious study of the refined asymptotics of the eigenvalues and eigenfunctions of the corresponding operator. The cases of continuous and summable potentials are considered.
\end{abstract}

Keywords and phrases: mixed problem, wave equation, graph, summable potential, Fourier method. AMS Subject Classification: 34B45, 35L05

1. Введение. Рассматривается смешанная задача для волнового уравнения на простейшим геометрическом графе $\Gamma=\left\{\gamma_{1}, \gamma_{2}\right\}$, состоящем из двух ребер, одно из которых $\gamma_{1}$ образует цикл. При параметризации каждого ребра графа отрезком $[0,1]$, задача будет иметь вид:

$$
\begin{gathered}
\frac{\partial^{2} u(x, t)}{\partial t^{2}}=\frac{\partial^{2} u(x, t)}{\partial x^{2}}-Q(x) u(x, t), \\
u_{1}(0, t)=u_{1}(1, t)=u_{2}(0, t), \quad u_{2}(1, t)=0,
\end{gathered}
$$

Работа выполнена при поддержке Российского научного фонда (проект № 19-11-00197). 


$$
\begin{gathered}
u_{1 x}^{\prime}(0, t)-u_{1 x}^{\prime}(1, t)+u_{2 x}^{\prime}(0, t)=0, \\
u(x, 0)=\varphi(x), \quad u_{t}^{\prime}(x, 0)=0, \quad x \in[0,1] .
\end{gathered}
$$

Здесь $u(x, t)=\left(u_{1}(x, t), u_{2}(x, t)\right)^{T}\left(T\right.$ - знак транспонирования), $\varphi(x)=\left(\varphi_{1}(x), \varphi_{2}(x)\right)^{T}, Q(x)=$ $\operatorname{diag}\left(q_{1}(x), q_{2}(x)\right), x \in[0,1], t \in(-\infty, \infty)$. Функции $q_{j}(x)$ и $\varphi_{j}(x)$ комплекснозначные (условия на $q_{j}(x)$ и $\varphi_{j}(x)$ будут сформулированы далее). Условия (2) обеспечивают непрерывность решения во внутреннем узле графа и неподвижное закрепление свободного конца на втором ребре. Условие (3) является частным случаем условий трансмиссии [11], нулевые значения для производных в (4) берутся для простоты.

Различные задачи для волнового уравнения на геометрических графах активно изучались: имеется достаточно много результатов в случае нулевого потенциала (см., например, $[12,14,16$, 20]), исследовались задачи с привлечением теории обобщенных функций (см. [13]), а также с помощью описания дифференциального уравнения в терминах производных по мере (см. [6]), проводилось численное решение задач (см. $[9,15])$. Исследовалась разрешимость и параболических смешанных задач на графе (например, в $[7,13])$.

На основе резольвентного подхода (см. $[3,5])$, в [2] получено классическое решение смешанной задачи на графе из двух ребер-колец, касающихся в одной точке (узле графа), в случае непрерывных потенциалов. При этом метод Фурье применялся с использованием идей по ускорению сходимости рядов, восходящих к А. Н. Крылову, и позволяющих получать классическое решение при минимальных требованиях на начальные функции. Для той же задачи в [19] дана краткая схема исследования в случае суммируемого потенциала. Наличие всего лишь двух ребер у графа хотя и упрощает техническую часть исследования, но позволяет понять трудности, возникающие при исследовании подобных задач. Так, в [1], где впервые на графе Г исследовалась смешанная задача для уравнения с инволюцией, показано, что простейшим может быть только рассматриваемый граф (на графе без цикла или с двумя циклами постановка задачи для уравнения первого порядка оказывается некорректной; см. [1]).

Здесь мы приводим полное доказательство существования решения задачи (1)-(4) при минимальных требованиях на начальные данные задачи, как в случае непрерывного, так и в случае суммируемого потенциала.

2. Схема резольвентного подхода. Следуя методу Фурье, полагаем в задаче $u_{1}(x, t)=T(t) y_{1}(x), u_{2}(x, t)=T(t) y_{2}(x)$ и приходим к спектральной задаче для оператора

$$
\begin{gathered}
(L y)(x)=\left(-y_{1}^{\prime \prime}(x)+q_{1}(x) y_{1}(x),-y_{2}^{\prime \prime}(x)+q_{2}(x) y_{2}(x)\right)^{T}, \quad y=\left(y_{1}, y_{2}\right)^{T}, \quad x \in[0,1], \\
y_{1}(0)=y_{1}(1)=y_{2}(0), \quad y_{2}(1)=0, \\
y_{1}^{\prime}(0)-y_{1}^{\prime}(1)+y_{2}^{\prime}(0)=0 .
\end{gathered}
$$

В дальнейшем также будет использоваться оператор $L_{0}$, который есть оператор $L$ при $q_{j}(x) \equiv 0$.

Приведем схему резольвентного подхода для смешанных задач, предложенную в [5]. Полученное по методу Фурье формальное решение задачи (1)-(4) представим в виде (см. также [17]):

$$
u(x, t)=-\frac{1}{2 \pi i} \int_{|\lambda|=r}\left(R_{\lambda} \varphi\right) \cos \rho t d \lambda-\sum_{n \geqslant n_{0}} \frac{1}{2 \pi i} \int_{\gamma_{n}}\left(R_{\lambda} \varphi\right) \cos \rho t d \lambda .
$$

Здесь $R_{\lambda}=(L-\lambda E)^{-1}$ - резольвента оператора $L$, a $r>0, n_{0}$ и контуры $\gamma_{n}$ одного и того же достаточно малого радиуса $\delta$ выбираются так, что все собственные значения $\lambda_{n}$ оператора $L$, начиная с некоторого $n_{0}$, по модулю превосходят $r$ и попадают только в $\gamma_{n}$ (возможность такого выбора обеспечивается имеющейся асимптотикой собственных значений), $\rho=\sqrt{\lambda}, \operatorname{Re} \rho \geqslant 0$.

В таком представлении формального решения совсем не участвуют собственные функции спектральной задачи, и для исследования необходимо знать только «грубую» асимптотику собственных значений и оценки для резольвенты оператора $L$. Отметим, что такой подход применим в задачах, где возникают трудности, связанные с возможной кратностью спектра (см., например, $[2,4,5])$. 
Преобразуем (5). Пусть комплексное число $\mu_{0}$ не является собственным значением операторов $L$ и $L_{0},\left|\mu_{0}\right|>r$, и $\mu_{0}$ находится вне $\gamma_{n}$ при $n \geqslant n_{0}$. Полагая $g=\left(L-\mu_{0} E\right) \varphi$, из (5) получим

$$
u(x, t)=-\frac{1}{2 \pi i}\left(\int_{\lambda \mid=r}+\sum_{n \geqslant n_{0}} \int_{\gamma_{n}}\right) \frac{R_{\lambda} g}{\lambda-\mu_{0}} \cos \rho t d \lambda .
$$

Дальнейшее преобразование приводит формальное решение $u(x, t)$ к виду:

$$
u(x, t)=u_{0}(x, t)+u_{1}(x, t),
$$

где

$$
\begin{aligned}
& u_{0}(x, t)=-\frac{1}{2 \pi i}\left[\int_{\lfloor\lambda \mid=r}+\sum_{n \geqslant n_{0}} \int_{\gamma_{n}}\right] \frac{R_{\lambda}^{0} g}{\lambda-\mu_{0}} \cos \rho t d \lambda, \\
& u_{1}(x, t)=-\frac{1}{2 \pi i}\left[\int_{\lfloor\lambda \mid=r}+\sum_{n \geqslant n_{0}} \int_{\gamma_{n}}\right] \frac{1}{\lambda-\mu_{0}}\left[R_{\lambda} g-R_{\lambda}^{0} g\right] \cos \rho t d \lambda,
\end{aligned}
$$

$R_{\lambda}^{0}$ - резольвента оператора $L_{0}$.

Исследуя резольвенты операторов $L$ и $L_{0}$, устанавливаем, что ряд $u_{1}(x, t)$ допускает почленное дифференцирование дважды по $x$ и $t$ за счет имеющихся оценок резольвент этих операторов. Ряд $u_{0}(x, t)$ оказывается формальным рядом задачи, соответствующей задаче $(1)-(4) \mathrm{c} q_{j}(x)=0$ и $\widetilde{\varphi}(x)=R_{\mu_{0}}^{0} g$ вместо $\varphi(x)$, и называемой эталонной:

$$
\begin{gathered}
\frac{\partial^{2} u(x, t)}{\partial t^{2}}=\frac{\partial^{2} u(x, t)}{\partial x^{2}}, \\
u_{1}(0, t)=u_{1}(1, t)=u_{2}(0, t), \quad u_{2}(1, t)=0, \\
u_{1 x}^{\prime}(0, t)-u_{1 x}^{\prime}(1, t)+u_{2 x}^{\prime}(0, t)=0, \\
u(x, 0)=\widetilde{\varphi}(x), \quad u_{t}^{\prime}(x, 0)=0, \quad x \in[0,1] .
\end{gathered}
$$

Решение такой задачи и, соответственно, $u_{0}(x, t)$, дается точной формулой, что позволяет судить о гладкости $u_{0}(x, t)$, не прибегая к дважды почленному дифференцированию ряда.

3. Случай непрерывного потенциала. Сначала рассмотрим задачу $(1)-(4)$ при $q_{j}(x) \in$ $C[0,1]$. Будем искать классическое решение задачи, т.е. функцию $u(x, t)=\left(u_{1}(x, t), u_{2}(x, t)\right)^{T}$ с дважды непрерывно дифференцируемыми по $x$ и $t$ компонентами, удовлетворяющую уравнению (1), краевым и начальным условиям (2)-(4). Для функции $\varphi(x)=\left(\varphi_{1}(x), \varphi_{2}(x)\right)^{T}$ требуем выполнения следующих условий: функции $\varphi_{j}(x) \in C^{2}[0,1]$ комплекснозначны, причем

$$
\begin{gathered}
\varphi_{1}(0)=\varphi_{1}(1)=\varphi_{2}(0), \quad \varphi_{2}(1)=0, \quad \varphi_{1}^{\prime}(0)-\varphi_{1}^{\prime}(1)+\varphi_{2}^{\prime}(0)=0, \\
\varphi_{1}^{\prime \prime}(0)-\varphi_{1}^{\prime \prime}(1)-\varphi_{1}(0)\left(q_{1}(0)-q_{1}(1)\right)=0, \\
\varphi_{1}^{\prime \prime}(0)-\varphi_{2}^{\prime \prime}(0)-\varphi_{1}(0)\left(q_{1}(0)-q_{2}(0)\right)=0, \quad \varphi_{2}^{\prime \prime}(1)=0 .
\end{gathered}
$$

Условия на $\varphi(x)$ являются минимальными для существования классического решения и следуют из постановки задачи (условия (14) очевидны, а (15) следуют из (1) и (14)).

3.1. Спектральная задача и резольвента оператора. Исследуем спектральную задачу $L y=\lambda y$, $y=y(x)=\left(y_{1}(x), y_{2}(x)\right)^{T}$ для оператора $L$.

Положим $\lambda=\rho^{2}, \operatorname{Re} \rho \geqslant 0$. Обозначим через $\left\{y_{j 1}(x), y_{j 2}(x)\right\},(j=1,2)$ фундаментальные системы решений уравнений

$$
y_{j}^{\prime \prime}(x)-q_{j}(x) y_{j}(x)+\rho^{2} y_{j}(x)=0, \quad j=1,2,
$$

с начальными условиями

$$
y_{j 1}(0)=1, \quad y_{j 1}^{\prime}(0)=0, \quad y_{j 2}(0)=0, \quad y_{j 2}^{\prime}(0)=1 .
$$


Тогда $y_{i j}(x)$ - целые функции по $\rho$ и по $\lambda$. Стандартными рассуждениями (см. [10, с. 74-75]) можно показать, что все $\rho$, для которых $\lambda=\rho^{2}$ являются собственными значениями оператора $L$, находятся в полуполосе $S=\{\rho|\operatorname{Re} \rho \geqslant 0,| \operatorname{Im} \rho \mid \leqslant h\}$, где $h>0$ и достаточно велико.

Лемма 1 (см. [10, с. 58-62]). В области $S$ справедливы асимптотические формуль

$$
\begin{aligned}
& y_{j 1}(x)=\cos \rho x+O\left(\rho^{-1}\right), \quad y_{j 1}^{\prime}(x)=-\rho \sin \rho x+O(1), \\
& y_{j 2}(x)=\frac{\sin \rho x}{\rho}+O\left(\rho^{-2}\right), \quad y_{j 2}^{\prime}(x)=\cos \rho x+O\left(\rho^{-1}\right) .
\end{aligned}
$$

Лемма 2 (см. [8], с. 17, 23). Для $y_{i j}(x)$ имеют место формуль

$$
y_{j 1}(x)=\cos \rho x+\int_{0}^{x} K_{j 1}(x, \xi) \cos \rho \xi d \xi, \quad y_{j 2}(x)=\frac{\sin \rho x}{\rho}+\int_{0}^{x} K_{j 2}(x, \xi) \frac{\sin \rho \xi}{\rho} d \xi,
$$

где функиии $K_{j k}(x, \xi)$ непрерывно дифференцируемы по $x$ и $\xi$, причем $K_{j 2}(x, 0) \equiv 0$.

Подставляя линейную комбинацию $y_{j 1}$ и $y_{j 2}$ в краевые условия, получим, что собственные значения оператора $L$ есть $\lambda=\rho^{2}, \rho$ - нули определителя

$$
\Delta(\rho)=\operatorname{det}\left(a_{k 1}, a_{k 2}, b_{k 1}, b_{k 2}\right)_{k=1}^{4},
$$

где $a_{11}=1-y_{11}(1), a_{12}=-y_{12}(1), b_{11}=b_{12}=0, a_{21}=1, a_{22}=0, b_{21}=-1, b_{22}=0, a_{31}=a_{32}=0$, $b_{31}=y_{21}(1), b_{32}=y_{22}(1), a_{41}=-y_{11}^{\prime}(1), a_{42}=1-y_{12}^{\prime}(1), b_{41}=0, b_{42}=1$.

Всюду далее соответствующие обозначения для оператора $L_{0}$ будем отмечать верхним индексом ${ }^{0}$. Имеем:

$$
\begin{aligned}
& y_{j 1}^{0}(x)=\cos \rho x, \quad y_{j 1}^{0}{ }^{\prime}(x)=-\rho \sin \rho x, \\
& y_{j 2}^{0}(x)=\frac{\sin \rho x}{\rho}, \quad y_{j 2}^{0}{ }^{\prime}(x)=\cos \rho x .
\end{aligned}
$$

Вычислив непосредственно $\Delta_{0}(\rho)$,

$$
\Delta_{0}(\rho)=-i \rho\left(1-e^{\rho i}\right)\left(1+e^{\rho i}\right)\left(3 e^{\rho i}+3 e^{-\rho i}-4\right),
$$

и учитывая, что собственные значения $\lambda_{n}$ оператора $L$ асимптотически приближаются к $\lambda_{n}^{0}$ при больших $n$, получим следующее утверждение.

Лемма 3. Собственные значения оператора $L_{0}$ равны $\lambda_{n}^{(0 k)}=\left(\rho_{n}^{(0 k)}\right)^{2}$, где

$$
\rho_{n}^{(01)}=\pi n, \quad \rho_{n}^{(02)}=b_{1}+2 \pi n, \quad \rho_{n}^{(03)}=b_{2}+2 \pi n, \quad b_{1,2}=-i \ln ((2 \pm \sqrt{5} i) / 3)
$$

(под $\ln z$ понимается главное значение $\operatorname{Ln} z$, при $\arg z \in(-\pi ; \pi])$. Собственные значения оператора L образуют три серии: $\lambda_{n}^{(k)}=\left(\rho_{n}^{(k)}\right)^{2}$ с асимптотикой

$$
\rho_{n}^{(k)}=\rho_{n}^{(0 k)}+\varepsilon_{n}^{(k)}, \quad k=1,2,3,
$$

где $\varepsilon_{n}^{(k)}=o(1), n \geqslant n_{0}, n_{0}$ - некоторое достаточно большое натуральное число.

Исследуем резольвенту оператора $L$.

Теорема 1. Для резольвенты $R_{\lambda}=\left(R_{1 \lambda}, R_{2 \lambda}\right)^{T}$ оператора $L$ имеет место формула

$$
R_{j \lambda} f(x)=\left(M_{j \rho} f_{j}\right)(x)+\Omega_{j \lambda}(x, f), \quad j=1,2, \quad f(x)=\left(f_{1}(x), f_{2}(x)\right)^{T},
$$

где

$$
\begin{gathered}
\left(M_{j \rho} f_{j}\right)(x)=\int_{0}^{x} M_{j}(x, \xi, \rho) f_{j}(\xi) d \xi, \quad M_{j}(x, \xi, \rho)=\left|\begin{array}{ll}
y_{j 1}(\xi) & y_{j 2}(\xi) \\
y_{j 1}(x) & y_{j 2}(x)
\end{array}\right| \\
\Omega_{j \lambda}(x, f)=\frac{y_{j 1}(x)}{\Delta(\rho)} \sum_{k=1}^{4} d_{k} \Delta_{k, 2 j-1}(\rho)+\frac{y_{j 2}(x)}{\Delta(\rho)} \sum_{k=1}^{4} d_{k} \Delta_{k, 2 j}(\rho) .
\end{gathered}
$$


Здесъ $\Delta_{k, s}(\rho)$ - алгебрачческие дополнения определителя $\Delta(\rho)$,

$$
d_{1}=\left.\left(M_{1 \rho} f_{1}\right)\right|_{x=1}, \quad d_{2}=0, \quad d_{3}=-\left.\left(M_{2 \rho} f_{2}\right)\right|_{x=1}, \quad d_{4}=\left.\int_{0}^{1} \frac{d}{d x} M_{1}(x, \xi, \rho)\right|_{x=1} f_{1}(\xi) d \xi .
$$

Доказательство. Для компонент вектора $y=\left(y_{1}, y_{2}\right)^{T}=R_{\lambda} f$ имеем дифференциальные уравнения

$$
y_{j}^{\prime \prime}(x)-q_{j}(x) y_{j}(x)+\rho^{2} y_{j}(x)=-f_{j}(x), \quad j=1,2,
$$

общие решения которых суть

$$
\begin{aligned}
& y_{1}(x)=c_{1} y_{11}(x)+c_{2} y_{12}(x)+\left(M_{1 \rho} f_{1}\right)(x), \\
& y_{2}(x)=b_{1} y_{21}(x)+b_{2} y_{22}(x)+\left(M_{2 \rho} f_{2}\right)(x) .
\end{aligned}
$$

Подставив $y_{k}(x)$ в краевые условия для оператора $L$, получим систему относительно $\left(c_{1}, c_{2}, b_{1}, b_{2}\right)^{T}$ с правой частью $\left(d_{1}, d_{2}, d_{3}, d_{4}\right)^{T}$ и матрицей $V(\rho)=\left(a_{k 1}, a_{k 2}, b_{k 1}, b_{k 2}\right)_{k=1}^{4}$, решая которую, придем к утверждению теоремы.

Для дальнейшего нам будет удобен иной вид $\Omega_{j \lambda}(x, f)$.

Лемма 4. Имеют место формуль

$$
\Omega_{j \lambda}(x, f)=v_{j 1}(x)\left(f_{1}, y_{11}\right)+v_{j 2}(x)\left(f_{1}, y_{12}\right)+v_{j 3}(x)\left(f_{2}, y_{21}\right)+v_{j 4}(x)\left(f_{2}, y_{22}\right), \quad j=1,2,
$$

где

$$
\begin{aligned}
& v_{11}(x)=v_{11}(x, \rho)=\sum_{k=1}^{2} \frac{y_{1 k}(x)}{\Delta(\rho)}\left[\Delta_{1 k}(\rho) y_{12}(1)+\Delta_{4 k}(\rho) y_{12}^{\prime}(1)\right] \\
& v_{12}(x)=v_{12}(x, \rho)=\sum_{k=1}^{2} \frac{y_{1 k}(x)}{\Delta(\rho)}\left[-\Delta_{1 k}(\rho) y_{11}(1)-\Delta_{4 k}(\rho) y_{11}^{\prime}(1)\right], \\
& v_{13}(x)=v_{13}(x, \rho)=-\sum_{k=1}^{2} \frac{y_{1 k}(x)}{\Delta(\rho)} \Delta_{3 k}(\rho) y_{22}(1), \\
& v_{14}(x)=v_{14}(x, \rho)=\sum_{k=1}^{2} \frac{y_{1 k}(x)}{\Delta(\rho)} \Delta_{3 k}(\rho) y_{21}(1),
\end{aligned}
$$

a $v_{2 j}(x)$ получаются аналогично с заменой $\Delta_{k 1}$ и $\Delta_{k 2}$ на $\Delta_{k 3}$ и $\Delta_{k 4}, y_{11}(x)$ и $y_{12}(x)$ на $y_{21}(x)$ и $y_{22}(x)$ соответственно,

$$
(f, g)=\int_{0}^{1} f(x) g(x) d x
$$

Доказательство. Вычислим явно $d_{k}$ из теоремы 1:

$$
\begin{array}{ll}
d_{1}=-y_{11}(1)\left(y_{12}, f_{1}\right)+y_{12}(1)\left(y_{11}, f_{1}\right), & d_{2}=0, \\
d_{3}=y_{21}(1)\left(y_{22}, f_{2}\right)-y_{22}(1)\left(y_{21}, f_{2}\right), & d_{4}=-y_{11}^{\prime}(1)\left(y_{12}, f_{1}\right)+y_{12}^{\prime}(1)\left(y_{11}, f_{1}\right) .
\end{array}
$$

Подставив эти выражения в $\Omega_{j \lambda}(x, f)$, получим требуемые соотношения.

Для резольвенты $R_{\lambda}^{0}=\left(R_{1 \lambda}^{0}, R_{2 \lambda}^{0}\right)^{T}$ имеет место формула

$$
R_{j \lambda}^{0} f(x)=\left(M_{j \rho}^{0} f_{j}\right)(x)+\Omega_{j \lambda}^{0}(x, f), \quad f=\left(f_{1}, f_{2}\right)^{T}, \quad(j=1,2),
$$

где $\left(M_{j \rho}^{0} f_{j}\right)(x), \Omega_{j \lambda}^{0}(x, f)$ определяются так же, как в теореме 1 и лемме 4 через $y_{k j}^{0}(x), \Delta_{k j}^{0}(\rho)$. При этом, учитывая вид $y_{i j}^{0}(x)$, получим следующее утверждение. 
Лемма 5. Для $\Omega_{\lambda}^{0}(x, f)=\left(\Omega_{1 \lambda}^{0}(x, f), \Omega_{2 \lambda}^{0}(x, f)\right)^{T}$ имеет место формула

$$
\Omega_{\lambda}^{0}(x, f)=N_{1}(f, \lambda) \cos \rho x+N_{2}(f, \lambda) \frac{\sin \rho x}{\rho},
$$

гde

$$
\begin{array}{ll}
N_{1}(f, \lambda)=\left(N_{11}, N_{12}\right)^{T}, & N_{2}(f, \lambda)=\left(N_{21}, N_{22}\right)^{T}, \\
N_{11}=\frac{1}{\Delta^{0}(\rho)} \sum_{j=1}^{4} d_{j}^{0} \Delta_{j 1}^{0}(\rho), & N_{12}=\frac{1}{\Delta^{0}(\rho)} \sum_{j=1}^{4} d_{j}^{0} \Delta_{j 3}^{0}(\rho), \\
N_{21}=\frac{1}{\Delta^{0}(\rho)} \sum_{j=1}^{4} d_{j}^{0} \Delta_{j 2}^{0}(\rho), & N_{22}=\frac{1}{\Delta^{0}(\rho)} \sum_{j=1}^{4} d_{j}^{0} \Delta_{j 4}^{0}(\rho) .
\end{array}
$$

3.2. Исследование компонент формального решения. Пусть $\delta>0$ и достаточно мало, чтобы контуры

где числа $\rho_{n}^{(0 k)}$ из леммы 3 , попадали в область

$$
\tilde{\gamma}_{n}^{k}=\left\{\rho|| \rho-\rho_{n}^{(0 k)} \mid=\delta\right\},
$$

$$
S=\{\rho|\operatorname{Re} \rho \geqslant 0,| \operatorname{Im} \rho \mid \leqslant h\},
$$

и $n_{0}$ выбираем так, чтобы все собственные значения $\rho_{n}^{(k)}$ при $n \geqslant n_{0}$ попадали внутрь $\tilde{\gamma}_{n}^{k}$. Обозначим через $\tilde{\gamma}_{n}$ объединение $\tilde{\gamma}_{n}^{k}, k=1,2,3$, а через $\gamma_{n}$ - образ $\tilde{\gamma}_{n}$ в $\lambda$-плоскости $\left(\lambda=\rho^{2}\right)$. Интегрирование по этим контурам используется в (5)-(9). Также введем обозначение $S_{\delta}=S \backslash \bigcup \tilde{\gamma}_{n}$.

Пусть

$$
\widetilde{\gamma}_{01}=\{\mu:|\mu|=\delta\}, \quad \widetilde{\gamma}_{02}=\left\{\mu:\left|\mu-b_{1}\right|=\delta\right\}, \quad \widetilde{\gamma}_{03}=\left\{\mu:\left|\mu-b_{2}\right|=\delta\right\} .
$$

Тогда, если $\rho \in \widetilde{\gamma}_{n}$, то $\rho=\pi n+\mu$, где $\mu \in \widetilde{\gamma}_{01}$ при нечетном $n$, и $\mu$ принадлежит объединению контуров $\widetilde{\gamma}_{0 k}, k=1,2,3$ при четном $n$. В дальнейшем через $\widetilde{\gamma}_{0}$ будем обозначать любой из этих контуров.

Из теоремы 1, учитывая, что $\left(M_{j \rho} f_{j}\right)(x),\left(M_{j \rho}^{0} f_{j}\right)(x)$ - целые, получим

$$
\begin{aligned}
& u_{0}(x, t)=-\frac{1}{2 \pi i}\left(\int_{\lambda \mid=r}+\sum_{n \geqslant n_{0}} \int_{\gamma_{n}}\right) \frac{\Omega_{\lambda}^{0}(x, g)}{\lambda-\mu_{0}} \cos \rho t d \lambda, \\
& u_{1}(x, t)=-\frac{1}{2 \pi i}\left(\int_{\lambda \mid=r}+\sum_{n \geqslant n_{0}} \int\right) \frac{1}{\lambda-\mu_{0}}\left[\Omega_{\lambda}(x, g)-\Omega_{\lambda}^{0}(x, g)\right] \cos \rho t d \lambda,
\end{aligned}
$$

где $\Omega_{\lambda}(x, g)=\left(\Omega_{1 \lambda}(x, g), \Omega_{2 \lambda}(x, g)\right)^{T}, \Omega_{\lambda}^{0}(x, g)=\left(\Omega_{1 \lambda}^{0}(x, g), \Omega_{2 \lambda}^{0}(x, g)\right)^{T}$.

Из леммы 1 и соотношений (16) непосредственным вычислением получаем следующие утверждения.

Лемма 6. При $\rho \in \widetilde{\gamma}_{n}$ имеем

$$
\Delta(\rho)=\Delta_{0}(\rho)+O\left(\rho^{-2}\right), \quad \frac{1}{\Delta(\rho)}=\frac{1}{\Delta_{0}(\rho)}\left[1+O\left(\rho^{-1}\right)\right], \quad\left|\Delta_{0}(\rho)\right| \geqslant c|\rho|^{-1} .
$$

Лемма 7. При $\rho \in \widetilde{\gamma}_{n}$ имеют место оценки

$$
\begin{array}{llll}
\Delta_{1 k}^{0}=O\left(\rho^{-1}\right), & \Delta_{3 k}^{0}=O\left(\rho^{-1}\right), & \Delta_{4 k}^{0}=O\left(\rho^{-2}\right), & k=1,3, \\
\Delta_{1 k}^{0}=O(1), & \Delta_{3 k}^{0}=O(1), & \Delta_{4 k}^{0}=O\left(\rho^{-1}\right), & k=2,4 .
\end{array}
$$

Лемма 8. При $\rho \in \widetilde{\gamma}_{n}$ имеют место оченки

$$
\begin{array}{llll}
\Delta_{1 k}=\Delta_{1 k}^{0}+O\left(\rho^{-2}\right), & \Delta_{3 k}=\Delta_{3 k}^{0}+O\left(\rho^{-2}\right), & \Delta_{4 k}=\Delta_{4 k}^{0}+O\left(\rho^{-3}\right), & k=1,3, \\
\Delta_{1 k}=\Delta_{1 k}^{0}+O\left(\rho^{-1}\right), & \Delta_{3 k}=\Delta_{3 k}^{0}+O\left(\rho^{-1}\right), & \Delta_{4 k}=\Delta_{4 k}^{0}+O\left(\rho^{-2}\right), & k=2,4 .
\end{array}
$$


При помощи лемм 7, 8 доказывается следующее утверждение.

Лемма 9. Если $\rho \in \widetilde{\gamma}_{n}, m o$

$$
\begin{array}{lll}
v_{s i}^{0^{(j)}}(x)=O\left(\rho^{j-1}\right), & v_{s i}{ }^{(j)}(x)=v_{s i}^{0}{ }^{(j)}(x)+O\left(\rho^{j-2}\right), & i=1,3, \\
v_{s i}^{0^{(j)}}(x)=O\left(\rho^{j}\right), & v_{s i}{ }^{(j)}(x)=v_{s i}^{0}{ }^{(j)}(x)+O\left(\rho^{j-1}\right), & i=2,4,
\end{array}
$$

где $s=1,2, j=0,1,2$ (у функиий $v_{s i}(x)$ аргумент $\rho$ для краткости опускаем).

Лемма 10 (см. [5, лемма 8]). Пусть $\beta_{n}(\mu)=(f(x, \mu), \psi(\pi n x))$, где $\psi(x)$ есть $\cos x$ или $\sin x$, $f(x, \mu)=f(x) \psi(\mu x), f(x) \in L_{2}[0,1] u \mu \in \gamma_{0}$. Тогда

$$
\sum\left|\beta_{n}(\mu)\right|^{2} \leqslant c\|f\|^{2}
$$

$\left(\|\cdot\|\right.$ - норма в $L_{2}[0,1]$, константа с не зависит от $\left.\mu \in \gamma_{0}\right)$, а кроме того, верна оченка

$$
\sum_{n=n_{1}}^{n_{2}} \frac{1}{n}\left|\beta_{n}(\mu)\right| \leqslant c \sqrt{\sum_{n=n_{1}}^{n_{2}} \frac{1}{n^{2}}}\|f\|,
$$

где $c>0$ и не зависит от $n_{1}, n_{2} u \mu \in \gamma_{0}$. Конечную сумму таких $\beta_{n}(\mu)$ будем обозначать $\widetilde{\beta}_{n}(\mu)$.

Лемма 11. При $\rho \in \widetilde{\gamma}_{n}, \rho=n \pi+\mu, \mu \in \widetilde{\gamma}_{0}$ имеют место оценки

$$
N_{1 j}=O\left(\rho^{-1} \widetilde{\beta}_{n}(\mu)\right), \quad N_{2 j}=O\left(\widetilde{\beta}_{n}(\mu)\right)
$$

Доказательство. Имеем

$$
\begin{aligned}
& \left(y_{k 1}^{0}(t), f(t)\right)=(f(t) \cos \mu t, \cos \pi n t)-(f(t) \sin \mu t, \sin \pi n t b n b n)=\widetilde{\beta}_{n}(\mu), \\
& \left(y_{k 2}^{0}(t), f(t)\right)=\rho^{-1}[(f(t) \cos \mu t, \cos \pi n t)-(f(t) \sin \mu t, \sin \pi n t)]=\rho^{-1} \widetilde{\beta}_{n}(\mu), \quad k=1,2 .
\end{aligned}
$$

Следовательно,

$$
d_{1}^{0}=O\left(\rho^{-1} \widetilde{\beta}_{n}(\mu)\right), \quad d_{3}^{0}=O\left(\rho^{-1} \widetilde{\beta}_{n}(\mu)\right), \quad d_{4}^{0}=O\left(\widetilde{\beta}_{n}(\mu)\right) .
$$

Учитывая, что в $S_{\delta}$ справедливы неравенство $\left|\Delta_{0}(\rho)\right| \geqslant c|\rho|^{-1}$ и оценки для $\Delta_{i j}^{0}(\rho)$ из леммы 7 , получим утверждение леммы.

Лемма 12 (см. [5], леммы 13, 14). Для $p(\xi) \in L_{2}[0,1]$ u $\rho=n \pi+\mu, \mu \in \widetilde{\gamma}_{0}$ uмеют место формульь

$$
\begin{aligned}
\left(p, y_{11}\right) & =\left(p_{1}(\xi) \cos \mu \xi, \cos n \pi \xi\right)-\left(p_{1}(\xi) \sin \mu \xi, \sin n \pi \xi\right), \\
\left(p, y_{11}-y_{11}^{0}\right) & =\frac{1}{n \pi+\mu}\left[\left(p_{2}(\xi) \cos \mu \xi, \cos n \pi \xi\right)-\left(p_{2}(\xi) \sin \mu \xi, \sin n \pi \xi\right)\right], \\
\left(p, y_{12}\right) & =\frac{1}{n \pi+\mu}\left[\left(p_{3}(\xi) \cos \mu \xi, \sin n \pi \xi\right)+\left(p_{3}(\xi) \sin \mu \xi, \cos n \pi \xi\right)\right], \\
\left(p, y_{12}-y_{12}^{0}\right) & =\frac{1}{(n \pi+\mu)^{2}}\left[\left(p_{4}(\xi) \cos \mu \xi, \cos n \pi \xi\right)-\left(p_{4}(\xi) \sin \mu \xi, \sin n \pi \xi\right)\right],
\end{aligned}
$$

где

$$
\begin{aligned}
& p_{1}(\xi)=p(\xi)+\int_{\xi}^{1} K_{11}(\tau, \xi) p(\tau) d \tau, \quad p_{2}(\xi)=p(\xi) K_{11}(\xi, \xi)-\int_{\xi}^{1} K_{11, \xi}^{\prime}(\tau, \xi) p(\tau) d \tau, \\
& p_{3}(\xi)=p(\xi)+\int_{\xi}^{1} K_{12}(\tau, \xi) p(\tau) d \tau, \quad p_{4}(\xi)=-p(\xi) K_{12}(\xi, \xi)+\int_{\xi}^{1} K_{12, \xi}^{\prime}(\tau, \xi) p(\tau) d \tau .
\end{aligned}
$$

Схожие утверждения имеют место и для $\left(p, y_{2 k}\right),\left(p, y_{2 k}-y_{2 k}^{0}\right), k=1,2$. 
3.3. Решение эталонной задачи. Здесь мы исследуем вектор-функцию $u_{0}(x, t)$ из $(8)$ и получим для нее точную формулу.

В силу тождества Гильберта $R_{\lambda}^{0} g-R_{\mu_{0}}^{0} g=\left(\lambda-\mu_{0}\right) R_{\lambda}^{0} R_{\mu_{0}}^{0} g$ имеем

$$
\frac{R_{\lambda}^{0} g}{\lambda-\mu_{0}}=\frac{\widetilde{\varphi}}{\lambda-\mu_{0}}+R_{\lambda}^{0} \widetilde{\varphi}
$$

где $\widetilde{\varphi}=R_{\mu_{0}}^{0} g$. Из (8) и (21) получаем следующее утверждение.

Лемма 13. Имеет место формула

$$
u_{0}(x, t)=-\frac{1}{2 \pi i} \int_{|\lambda|=r}\left(R_{\lambda}^{0} \widetilde{\varphi}\right) \cos \rho t d \lambda-\sum_{n \geqslant n_{0}} \frac{1}{2 \pi i} \int_{\tilde{\gamma}_{n}}\left(R_{\lambda}^{0} \widetilde{\varphi}\right) \cos \rho t d \lambda .
$$

Таким образом, $u_{0}(x, t)$ представляет собой формальное решение задачи (10)-(13). Несложно проверить, что $\widetilde{\varphi}=\left(\widetilde{\varphi}_{1}, \widetilde{\varphi}_{2}\right)$ удовлетворяет соотношениям (14) и

$$
\widetilde{\varphi}_{1}^{\prime \prime}(0)=\widetilde{\varphi}_{1}^{\prime \prime}(1)=\widetilde{\varphi}_{2}^{\prime \prime}(0), \quad \widetilde{\varphi}_{2}^{\prime \prime}(1)=0,
$$

и $\widetilde{\varphi}_{j}(x) \in C^{2}[0,1]$.

Учитывая (17) и формулы

$\cos \rho x \cos \rho t=\frac{1}{2}[\cos \rho(x+t)+\cos \rho(x-t)], \quad \sin \rho x \cos \rho t=\frac{1}{2}[\sin \rho(x+t)+\sin \rho(x-t)]$, получаем следующий факт.

Лемма 14. Имеет место формула

$$
\Omega_{\lambda}^{0}(x, f) \cos \rho t=\frac{1}{2}\left[\Omega_{\lambda}^{0}(x+t, f)+\Omega_{\lambda}^{0}(x-t, f)\right],
$$

где $\Omega_{\lambda}^{0}=\left(\Omega_{1 \lambda}^{0}, \Omega_{2 \lambda}^{0}\right)^{T}$.

Положим

$$
F(x)=-\frac{1}{2 \pi i}\left(\int_{|\lambda|=r}+\sum_{n \geqslant n_{0}} \int_{\gamma_{n}}\right) \frac{1}{\lambda-\mu_{0}} \Omega_{\lambda}^{0}(x, g) d \lambda .
$$

В силу (17) ряд в (24) определен при всех $x \in(-\infty, \infty)$.

Лемма 15. Рядъ

$$
-\frac{1}{2 \pi i}\left(\int_{\lambda \mid=r}+\sum_{n \geqslant n_{0}} \int_{\gamma_{n}}\right) \frac{1}{\lambda-\mu_{0}} \frac{d^{j}}{d x^{j}} \Omega_{\lambda}^{0}(x, g) d \lambda, \quad j=0,1,
$$

сходятся абсолютно и равномерно при $x \in(-\infty, \infty)$.

Доказательство. В силу лемм 5 и 11 при $\rho \in \widetilde{\gamma}_{n}$ имеем оценки:

$$
\begin{gathered}
\left|\Omega_{j \lambda}^{0}(x, g)\right| \leqslant \frac{c}{|\rho|}\left|\widetilde{\beta}_{n}(\mu)\right|, \quad\left|\frac{d}{d x} \Omega_{j \lambda}^{0}(x, g)\right| \leqslant c\left|\widetilde{\beta}_{n}(\mu)\right|, \\
\left|\int_{\gamma_{n}} \frac{\Omega_{j \lambda}^{0}(x, g)}{\lambda-\mu_{0}} d \lambda\right| \leqslant \frac{1}{n^{2}} \int_{\widetilde{\gamma}_{0}}\left|\widetilde{\beta}_{n}(\mu)\right||d \mu|, \quad\left|\int_{\gamma_{n}} \frac{\left(\Omega_{j \lambda}^{0}(x, g)\right)_{x}^{\prime}}{\lambda-\mu_{0}} d \lambda\right| \leqslant \frac{1}{n} \int_{\widetilde{\gamma}_{0}}\left|\widetilde{\beta}_{n}(\mu)\right||d \mu| .
\end{gathered}
$$

Отсюда с учетом (20) следует равномерная сходимость ряда (24) и почленно продифференцированного ряда.

Из (18) и лемм 14 и 15 получаем следующее утверждение.

Лемма 16. Имеет место формула

$$
u_{0}(x, t)=\frac{1}{2}(F(x+t)+F(x-t)) .
$$


Лемма 17. Для $F(x)=\left(F_{1}(x), F_{2}(x)\right)^{T}$ справедливы следующие утверждения:

(i) Eсли $x \in[0,1]$, mо $F(x)=\widetilde{\varphi}(x)=\left(R_{\mu_{0}}^{0} g\right)(x)$.

(ii) Для любых $x$ имеют место соотношения

$$
\begin{aligned}
F_{1}(-x) & =\frac{1}{3}\left[2 F_{1}(1-x)+2 F_{2}(x)-F_{1}(x)\right], \\
F_{2}(-x) & =\frac{1}{3}\left[2 F_{1}(x)+2 F_{1}(1-x)-F_{2}(x)\right], \\
F_{1}(1+x) & =\frac{1}{3}\left[2 F_{1}(x)+2 F_{2}(x)-F_{1}(1-x)\right], \\
F_{2}(1+x) & =-F_{2}(1-x) .
\end{aligned}
$$

(iii) $F_{k}(x) \in C^{2}(-\infty, \infty), k=1,2$.

Доказательство. 1. Из (18) при $t=0$ и $x \in[0,1]$ имеем

$$
u_{0}(x, 0)=-\frac{1}{2 \pi i}\left(\int_{\lambda \mid=r}+\sum_{n \geqslant n_{0}} \int_{\gamma_{n}}\right) \frac{1}{\lambda-\mu_{0}} \Omega_{\lambda}^{0}(x, g) d \lambda=F(x) .
$$

Так как для $\widetilde{\varphi}=R_{\mu_{0}}^{0} g$ справедливо $(21)$, то аналогично лемме 13 получаем, что при $x \in[0,1]$

$$
F(x)=-\frac{1}{2 \pi i}\left(\int_{\lambda \mid=r}+\sum_{n \geqslant n_{0}} \int_{\gamma_{n}}\right) R_{\lambda}^{0} \widetilde{\varphi} d \lambda,
$$

т.е. является рядом Фурье функции $\widetilde{\varphi}(x) \in D_{L_{0}}$ по собственным и присоединенным функциям оператора $L_{0}$. Отсюда следует первое утверждение леммы.

2. Подставляя (25) в (2)-(3), получим систему

$$
\begin{gathered}
F_{1}(t)+F_{1}(-t)=F_{1}(1+t)+F_{1}(1-t), \\
F_{1}(t)+F_{1}(-t)=F_{2}(t)+F_{2}(-t), \\
F_{2}(1+t)+F_{2}(1-t)=0, \\
F_{1}^{\prime}(t)+F_{1}^{\prime}(-t)-F_{1}^{\prime}(1-t)+F_{2}^{\prime}(t)+F_{2}^{\prime}(t)=0 .
\end{gathered}
$$

Интегрируя (34) и учитывая непрерывность $F_{k}(x)$, имеем

$$
F_{1}(t)-F_{1}(-t)-F_{1}(1+t)+F_{1}(1-t)+F_{2}(t)-F_{2}(-t)=0 .
$$

Из (31)-(33) и (35) придем к (26)-(29).

Отметим, что при $x \in[0,1]$ правая часть в (26)-(27) определена, а, значит, по соотношениям (26)-(27) функция $F(x)$ может быть продолжена на отрезок $[-1,0]$, а затем по (28)-(29) на отрезок $[1,2]$. Теперь при $x \in[1,2]$ правая часть в (26)-(27) определена, а, значит, по соотношениям $(26)-(27) F(x)$ продолжаем на отрезок $[-2,-1]$, а затем по $(28)-(29)$ на отрезок $[2,3]$. Таким образом, функция $F(x)$ оказывается продолженной с отрезка $[0,1]$ на всю ось аналитическим выражением.

3. Непрерывная дифференцируемость на всей оси функций $F_{k}(x)$ следует из леммы 15 . Далее, согласно утверждению (i) при $x \in[0,1] F(x)$ и $\tilde{\varphi}(x)$ совпадают, откуда сразу вытекает дважды дифференцируемость $F(x)$ на отрезке $[0,1]$. В силу (26)-(29) $F(x)$ дважды дифференцируема на всей оси, возможно, за исключением точек $x=k, k$-любое целое число. Покажем, что $F^{\prime \prime}(k-0)=$ $F^{\prime \prime}(k+0)$.

Так как $F_{k}^{\prime \prime}(0+0)=\tilde{\varphi}_{k}^{\prime \prime}(0), F_{k}^{\prime \prime}(1-0)=\tilde{\varphi}_{k}^{\prime \prime}(1)$, то для $F_{k}^{\prime \prime}(0+0)$ и $F_{k}^{\prime \prime}(1-0)$ справедливы соотношения (23). Отсюда, дифференцируя (26)-(29) дважды и переходя в полученных соотношениях к пределу при $x \rightarrow 0+0$ и $x \rightarrow 1-0$, получим

$$
F_{k}^{\prime \prime}(0-0)=F_{k}^{\prime \prime}(0+0), \quad F_{k}^{\prime \prime}(1-0)=F_{k}^{\prime \prime}(1+0) .
$$

В остальных точках $x=k, k \in \mathbb{Z}$ доказывается по индукции. 
Из лемм 16 и 17 вытекает следующее утверждение.

Теорема 2. Классическое решение задачи (10)-(13) существует и имеет вид

$$
u_{0}(x, t)=\frac{1}{2}(F(x+t)+F(x-t))
$$

где $F(x)=\left(F_{1}(x), F_{2}(x)\right)^{T}, F(x)$-дважды непрерывно дифференцируемая функция, причем $F(x)=\widetilde{\varphi}(x)=\left(R_{\mu_{0}}^{0} g\right)(x)$ при $x \in[0,1]$, а на всю ось $F(x)$ продолжсается с помощью соотношений (26)-(29).

3.4. Классическое решение. Исследуем теперь ряд $u_{1}(x, t)$.

Лемма 18. Ряд (19) и ряды, получающиеся из него почленным дифберенцированием по $x$ и $t$ до второго порядка, сходятся абсолютно и равномерно по $x \in[0,1] u t \in[-T, T]$, где $T>0$ любое фиксированное число.

Доказательство. Рассмотрим в (19) ряд $\sum_{n=n_{0}}^{\infty} b_{n}(x, t)$, где

$$
\begin{gathered}
b_{n}(x, t)=-\frac{1}{2 \pi i} \int_{\gamma_{n}} \frac{1}{\lambda-\mu_{0}}\left[\Omega_{\lambda}(x, g)-\Omega_{\lambda}^{0}(x, g)\right] \cos \rho t d \lambda=-\frac{1}{\pi i} \int_{\widetilde{\gamma}_{n}} \frac{\rho}{\rho^{2}-\mu_{0}} J(x, \rho) \cos \rho t d \rho, \\
J(x, \rho)=\Omega_{\lambda}(x, g)-\Omega_{\lambda}^{0}(x, g) .
\end{gathered}
$$

Согласно лемме 4 для компонент $J(x, \rho)$ и $J^{(j)}(x, \rho)=d^{j} J(x, \rho) / d x^{j}$ справедливо следующее представление:

$$
\begin{aligned}
& J_{k}^{(j)}(x, \rho)= v_{k 1}^{(j)}(x)\left(g_{1}, y_{11}\right)+v_{k 2}^{(j)}(x)\left(g_{1}, y_{12}\right)+v_{k 3}^{(j)}(x)\left(g_{2}, y_{21}\right)+v_{k 4}^{(j)}(x)\left(g_{2}, y_{22}\right)- \\
&-v_{k 1}^{0(j)}(x)\left(g_{1}, y_{11}^{0}\right)-v_{k 2}^{0(j)}(x)\left(g_{1}, y_{12}^{0}\right)-v_{k 3}^{0(j)}(x)\left(g_{2}, y_{21}^{0}\right)-v_{k 4}^{0(j)}(x)\left(g_{2}, y_{22}^{0}\right)= \\
&=\left[v_{k 1}^{(j)}(x)-v_{k 1}^{0(j)}(x)\right]\left(g_{1}, y_{11}\right)+\left[v_{k 2}^{(j)}(x)-v_{k 2}^{0(j)}(x)\right]\left(g_{1}, y_{12}\right)+\left[v_{k 3}^{(j)}(x)-v_{k 3}^{0(j)}(x)\right]\left(g_{2}, y_{21}\right)+ \\
&+\left[v_{k 4}^{(j)}(x)-v_{k 4}^{0(j)}(x)\right]\left(g_{2}, y_{22}\right)+v_{k 1}^{0(j)}(x)\left(g_{1}, y_{11}-y_{11}^{0}\right)+v_{k 2}^{0(j)}(x)\left(g_{1}, y_{12}-y_{12}^{0}\right)+ \\
& \quad+v_{k 3}^{0(j)}(x)\left(g_{2}, y_{21}-y_{21}^{0}\right)+v_{k 4}^{0(j)}(x)\left(g_{2}, y_{22}-y_{22}^{0}\right), \quad j=0,1,2 .
\end{aligned}
$$

Из лемм 9, 12 при $\rho \in \widetilde{\gamma}_{n}, \rho=\pi n+\mu, \mu \in \widetilde{\gamma}_{0}$ следуют оценки

$$
J(x, \rho)=O\left(\frac{1}{n^{2}} \tilde{\beta}_{n}(\mu)\right), \quad J_{x}^{\prime}(x, \rho)=O\left(\frac{1}{n} \tilde{\beta}_{n}(\mu)\right), \quad J_{x^{2}}^{\prime \prime}(x, \rho)=O\left(\tilde{\beta}_{n}(\mu)\right),
$$

откуда сразу получаем, что если $j=0,1$, то

$$
\left|b_{n, x^{j}}^{(j)}(x, t)\right|=O\left(n^{-2}\right) ;
$$

тем самым, ряды $\sum\left|b_{n, x^{j}}^{(j)}(x, t)\right|$ сходятся равномерно по $x \in[0,1]$ и $t \in[-T, T]$. Далее, из (36) и леммы 10 имеем

$$
\sum_{n=n_{1}}^{n_{2}}\left|b_{n, x^{2}}^{\prime \prime}(x, t)\right|=\int_{\tilde{\gamma}_{0}} \sum_{n=n_{1}}^{n_{2}} O\left(n^{-1} \widetilde{\beta}_{n}(\mu)\right)|d \mu|=\int_{\widetilde{\gamma}_{0}} O\left(\sqrt{\sum_{n=n_{1}}^{n_{2}} \frac{1}{n^{2}}}\right)|d \mu|=O\left(\sqrt{\sum_{n=n_{1}}^{n_{2}} \frac{1}{n^{2}}}\right),
$$

где оценка $O(\ldots)$ равномерна по $x \in[0,1]$ и $t \in[-T, T]$. Отсюда следует равномерная сходимость ряда $\sum b_{n, x^{2}}^{\prime \prime}(x, t)$. Аналогично исследуются ряды $\sum b_{n, t^{j}}^{(j)}(x, t)$.

Теорема 3. Eсли $q_{j}(x) \in C[0,1], \varphi_{j}(x) \in C^{2}[0,1]$ и $\varphi_{j}(x)$ удовлетворяют условиям (14)-(15), то формальное решение $и(x, t)$ есть классическое решение задачи (1)-(4).

Доказательство. Из (6) и теоремы 1 для формального решения $u(x, t)$ имеем:

$$
u(x, t)=-\frac{1}{2 \pi i}\left(\int_{\lambda \mid=r}+\sum_{n \geqslant n_{0}} \int_{\gamma_{n}}\right) \frac{\Omega_{\lambda}(x, g)}{\lambda-\mu_{0}} \cos \rho t d \lambda,
$$


откуда с учетом леммы 4 и оценок из лемм 8, 9 следует, что этот ряд и ряды, образованные из него почленным дифференцированием один раз по $x$ и $t$, сходятся абсолютно и равномерно. Поэтому $u(x, t)$ удовлетворяет начальным и граничным условиям.

Далее, в силу теоремы 2 и леммы $18 u(x, t)$, преобразованная к виду (7), дважды непрерывно дифференцируема. Докажем, что $u(x, t)$ из $(7)$ удовлетворяет уравнению (1). Рассмотрим оператор

$$
M=\frac{\partial^{2}}{\partial t^{2}}-\frac{\partial^{2}}{\partial x^{2}} .
$$

Согласно теореме 2 имеем $M u_{0}(x, t)=0$. Далее, так как $\left(R_{\lambda} g\right)^{\prime \prime}=Q(x) R_{\lambda} g-\rho^{2} R_{\lambda} g-g$, то для слагаемых ряда $u_{1}(x, t)$ получим

$$
M\left(-\frac{1}{2 \pi i} \int_{\gamma} \frac{R_{\lambda} g-R_{\lambda}^{0} g}{\lambda-\mu_{0}} \cos \rho t d \lambda\right)=-Q(x) \frac{1}{2 \pi i} \int_{\gamma} \frac{R_{\lambda} g}{\lambda-\mu_{0}} \cos \rho t d \lambda=-Q(x) \frac{1}{2 \pi i} \int_{\gamma} R_{\lambda} \varphi \cos \rho t d \lambda
$$

(здесь $\gamma$ один из контуров $\gamma_{n}$ или $\left.|\lambda|=r\right)$, откуда $M\left(u_{0}(x, t)+u_{1}(x, t)\right)=-Q(x) u(x, t)$.

4. Случай суммируемого потенциала. Исследуем теперь задачу (1)-(4) в предположении, что $q_{j}(x) \in L[0,1]$ и комплекснозначны. Будем использовать технику и некоторые результаты работ $[4,18]$.

Классическим решением теперь будем называть вектор-функцию $u(x, t)=\left(u_{1}(x, t), u_{2}(x, t)\right)^{T}$, компоненты которой абсолютно непрерывны вместе с первой производной по $x$ и $t$, удовлетворяют граничным и начальным условиям (2)-(4) и удовлетворяют дифференциальному уравнению (1) лишь почти всюду.

Минимальные условия на $\varphi(x)$ следующие: компоненты $\varphi(x), \varphi^{\prime}(x)$ абсолютно непрерывны, $L \varphi \in L_{2}^{2}[0,1]$, и выполнены условия (14).

Снова преобразуем ряд формального решения с помощью (6)-(9). Теорема 1, а также леммы 1 , 4-8 сохраняются. Функция $g=L \varphi-\mu_{0} \varphi$ теперь из $L_{2}^{2}[0,1]$.

Для $u_{0}(x, t)$ справедливо представление $(25)$, где $F(x)$ определяется в $(24)$, но вместо леммы 17 теперь имеем следующее утверждение.

Лемма 19. Вектор-функции $F(x)$ u $F^{\prime}(x)$ абсолютно непреръьвнь при $x \in(-\infty,+\infty)$, $F^{\prime \prime}(x) \in L_{2}^{2}[-A, A]$ для любого $A>0, F(x)$ удовлетворяет соотношениям (26)-(29), причем $F(x)=\tilde{\varphi}(x)=R_{\mu_{0}}^{0} g$ при $x \in[0,1]$.

Доказательство. Так как $\tilde{\varphi}(x)=R_{\mu_{0}}^{0} g$, а $g \in L_{2}^{2}[0,1]$, то из соотношений (26)-(29), справедливых в силу (25), утверждение леммы очевидно.

Теорема 4. Пусть $u_{0}(x, t)=\frac{1}{2}(F(x+t)+F(x-t))$, где функиия $F(x)$ такая же, как в лемме 19. Тогда $u_{0}(x, t)$ непреръвно дифберениируема по $x \in[0,1] u t \in(-\infty,+\infty) ; u_{0 x}^{\prime}(x, t), u_{0 t}^{\prime}(x, t)$ абсолютно непрерывны по $x$ u $t$ соответственно; производные $\frac{\partial^{2} u_{0}(x, t)}{\partial t^{2}}, \frac{\partial^{2} u_{0}(x, t)}{\partial x^{2}}$ конечны почти при всех $x$ и $t$, при этом почти всюду выполнено (10); для $u_{0}(x, t)$ справедливы соотношения (11)-(13). Таким образом и $(x, t)$ есть решение задачи (10)-(13), когда уравнение (10) удовлетворяется почти всюду.

Доказательство. Согласно лемме 19 производная $F^{\prime \prime}(x)$ существует почти всюду на $(-\infty,+\infty)$. Отсюда так же, как в [18, лемма 6], доказывается, что производные $\frac{\partial^{2} u_{0}(x, t)}{\partial t^{2}}, \frac{\partial^{2} u_{0}(x, t)}{\partial x^{2}}$ существуют почти всюду в $Q_{T}=[0,1] \times[-T, T], T>0$ и любое, и для таких $x$ и $t$ они совпадают. Остальные утверждения легко следуют из соотношения (25) и леммы 19.

Вместо леммы 2 о представлении $y_{i j}(x)$ через операторы преобразования справедлива следующая лемма. 
Лемма 20 (см. [18, лемма 7]; [4, лемма 2]). Если $|\operatorname{Jm} \rho| \leqslant h, m o$

$$
\begin{aligned}
& y_{j 1}(x, \rho)=\cos \rho x+\frac{1}{2 \rho} \sin \rho x \int_{0}^{x} q_{j}(\tau) d \tau+\frac{1}{4 \rho} \int_{0}^{x}\left[q_{j}\left(\frac{x-\tau}{2}\right)+q_{j}\left(\frac{x+\tau}{2}\right)\right] \sin \rho \tau d \tau+O\left(\rho^{-2}\right), \\
& y_{j 2}(x, \rho)=\frac{\sin \rho x}{\rho}+\frac{1}{2 \rho^{2}} \cos \rho x \int_{0}^{x} q_{j}(\tau) d \tau+\frac{1}{4 \rho^{2}} \int_{0}^{x}\left[q_{j}\left(\frac{x-\tau}{2}\right)+q_{j}\left(\frac{x+\tau}{2}\right)\right] \cos \rho \tau d \tau+O\left(\rho^{-3}\right),
\end{aligned}
$$

где оценки $O(\ldots)$ равномерны по $x \in[0,1]$.

Далее лемма 9 заменяется на следующее утверждение.

Лемма 21. Если $\rho \in \widetilde{\gamma}_{n}$, mo

$$
\begin{aligned}
& v_{s i}^{0(j)}(x)=O\left(\rho^{j-1}\right), \quad i=1,3, \quad v_{s i}^{0(j)}(x)=O\left(\rho^{j}\right), \quad i=2,4, \quad s=1,2, \quad j=0,1,2, \\
& v_{s 1}^{(j)}(x, \rho)=v_{s 1}^{0}{ }^{(j)}(x, \rho)+O\left(\rho^{j-2}\right) \quad(j=0,1), \\
& v_{s 2}^{(j)}(x, \rho)=v_{s 2}^{0}{ }^{(j)}(x, \rho)+O\left(\rho^{j-1}\right) \quad(j=0,1), \\
& v_{s 3}^{(j)}(x, \rho)=v_{s 3}^{0(j)}(x, \rho)+O\left(\rho^{j}\right) \quad(j=0,1), \\
& v_{s 4}^{(j)}(x, \rho)=v_{s 4}^{0}{ }^{(j)}(x, \rho)+O\left(\rho^{j-1}\right) \quad(j=0,1), \\
& v_{s 1}^{\prime \prime}(x, \rho)-q_{s}(x) v_{s 1}(x, \rho)-v_{s 1}^{0}{ }^{\prime \prime}(x, \rho)=O(1), \\
& v_{s 2}^{\prime \prime}(x, \rho)-q_{s}(x) v_{s 2}(x, \rho)-v_{s 2}^{0 \prime \prime}(x, \rho)=O(\rho) \text {, } \\
& v_{s 3}^{\prime \prime}(x, \rho)-q_{s}(x) v_{s 3}(x, \rho)-v_{s 3}^{0}{ }^{\prime \prime}(x, \rho)=O\left(\rho^{2}\right) \text {, } \\
& v_{s 4}^{\prime \prime}(x, \rho)-q_{s}(x) v_{s 4}(x, \rho)-v_{s 4}^{0}{ }^{\prime \prime}(x, \rho)=O(\rho),
\end{aligned}
$$

$(s=1,2)$, где производные берутся по $x$ и оченки $O(\ldots)$ равномерны по $x \in[0,1]$ (в последних четырех равенствах $O(\ldots)$ означает, что $\left.\|O(\omega)\|_{\infty} \leqslant c|\omega|\right)$.

Доказательство. Оценки для $v_{k i}^{0(j)}(x)$ очевидны. Оценки для $v_{k i}^{(j)}(x, \rho)$ при $j=0,1$ следуют из лемм 7,8 . Последние оценки имеют место в силу того, что $v_{k i}^{\prime \prime}(x, \rho)-q_{k}(x) v_{k i}(x, \rho)=-\rho^{2} v_{k i}(x, \rho)$ и оценок для $v_{k i}(x, \rho)$.

Приведем теперь необходимые результаты из [18]. Из [18, лемма 8] сразу получаем следующий факт.

Лемма 22. Обозначим через $p(x)$ одну из функиий

$$
\int_{x}^{1} m(\xi) q\left(\frac{\xi-x}{2}\right) d \xi \quad \text { или } \int_{x}^{1} m(\xi) q\left(\frac{\xi+x}{2}\right) d \xi,
$$

где $m(\xi)$ либо $g_{1}(\xi)$, либо $g_{2}(\xi)\left(g_{k}(x)\right.$ - компоненты вектора $\left.g=\left(L-\mu_{0} E\right) \varphi\right)$, а $q(x)$ - это либо $q_{1}(x)$, либо $q_{2}(x)$. Тогда

где $\|\cdot\|_{L_{s}}-$ норма в $L_{s}[0,1]$.

$$
\|p\|_{L_{2}} \leqslant 2\|m\|_{L_{2}} \cdot\|q\|_{L_{1}},
$$

Отметим, что лемма 10 справедлива, если $f(x)$ одна из функций

$$
g_{j}(x), \quad g_{j}(x) \int_{0}^{x} q_{s}(\xi) d \xi,
$$

а в силу леммы 22 , если $f(x)$ одна из функций $p(x)$ из леммы 22. Кроме того,

$$
\sum_{n=n_{1}}^{n_{2}} \frac{1}{n} \widetilde{\beta}_{n}(\mu) \leqslant c \sqrt{\sum_{n=n_{1}}^{n_{2}} \frac{1}{n^{2}}}\|g\|_{2},
$$


где $c>0$ - константа, не зависящая от $n_{1}, n_{2}, \mu \in \gamma_{0}$, и $\|g\|_{2}$ теперь обозначает норму векторфункции $g(x)=\left(g_{1}(x), g_{2}(x)\right)^{T}$ в $L_{2}^{2}[0,1]$.

Аналогично [18, лемма 9] (см. также лемму 12) из леммы 20 получаем следующее утверждения.

Лемма 23. Eсли $g(x)=\left(g_{1}(x), g_{2}(x)\right)^{T} \in L_{2}^{2}[0,1], \rho=n \pi+\mu, \mu \in \gamma_{0}$, то имеют место формуль

$$
\begin{gathered}
\left(g_{s}, y_{j 1}\right)=O\left(\widetilde{\beta}_{n}(\mu)\right)+O\left(\rho^{-1} \widetilde{\beta}_{n}(\mu)\right)+O\left(\rho^{-2}\|g\|_{2}\right), \\
\left(g_{s}, y_{j 1}-y_{j 1}^{0}\right)=O\left(\rho^{-1} \widetilde{\beta}_{n}(\mu)\right)+O\left(\rho^{-2}\|g\|_{2}\right), \\
\left(g_{s}, y_{j 2}\right)=O\left(\rho^{-1} \widetilde{\beta}_{n}(\mu)\right)+O\left(\rho^{-2} \widetilde{\beta}_{n}(\mu)\right)+O\left(\rho^{-3}\|g\|_{2}\right), \\
\left(g_{s}, y_{j 2}-y_{j 2}^{0}\right)=O\left(\rho^{-2} \widetilde{\beta}_{n}(\mu)\right)+O\left(\rho^{-3}\|g\|_{2}\right),
\end{gathered}
$$

где $j=1,2, s=1,2$.

Из лемм 21-23 получаем следующее утверждение.

Лемма 24. Если $\rho=\pi n+\mu, \mu \in \tilde{\gamma}_{0}, \Omega_{\lambda}(x, g)=\left(\Omega_{1 \lambda}(x, g), \Omega_{2 \lambda}(x, g)\right)^{T}$, mo

$$
\frac{d^{j}}{d x^{j}}\left(\Omega_{\lambda}(x, g)\right)=O\left(\rho^{j-1} \widetilde{\beta}_{n}(\mu)\right)+O\left(\rho^{j-2}\|g\|_{2}\right), \quad(j=0,1)
$$

и аналогичные оченки для $\frac{d^{j}}{d x^{j}}\left(\Omega_{\lambda}^{0}(x, g)\right), u$

$$
\frac{d^{j}}{d x^{j}}\left(\Omega_{\lambda}(x, g)-\Omega_{\lambda}^{0}(x, g)\right)=O\left(\rho^{j-2} \widetilde{\beta}_{n}(\mu)\right)+O\left(\rho^{j-3}\|g\|_{2}\right), \quad j=0,1 .
$$

Отсюда и из леммы 10, как и в [18, Лемма 12, Теорема 5], вытекают следующие леммы.

Лемма 25. Ряд $u_{1}(x, t)$ и ряды, полученные из $u_{1}(x, t)$ почленным дифберениированием по $x$ и дважды по $t$, сходятся абсолютно и равномерно в $Q_{T}=[0,1] \times[-T, T]$, при любом $T>0$.

Лемма 26. Функция $u_{1, x}^{\prime}(x, t)$ абсолютно непрерывна по $x$, и почти всюду справедливо соотношение

$$
u_{1, x^{2}}^{\prime \prime}(x, t)=Q(x) u_{1}(x, t)+d(x, t)
$$

Здесъ $Q(x)=\operatorname{diag}\left(q_{1}(x), q_{2}(x)\right)$,

$$
d(x, t)=-\frac{1}{2 \pi i}\left(\int_{\lambda \mid=r}+\sum_{n \geqslant n_{0}} \int_{\gamma_{n}}\right) \frac{\lambda}{\lambda-\mu_{0}}\left[\Omega_{\lambda}(x, g)-\Omega_{\lambda}^{0}(x, g)\right] \cos \rho t d \lambda,
$$

ряд d $(x, t)$ абсолютно и равномерно сходится в $Q_{T}$.

Теперь из лемм 19, 25, 26 получаем (аналогично [18, Теорема 5]) окончательный результат.

Теорема 5. Если $q_{j}(x) \in L[0,1]$, вектор-функиии $\varphi(x)$ и $\varphi^{\prime}(x)$ абсолютно непрерывны, $L \varphi \in$ $L_{2}^{2}[0,1], u \varphi(x)$ удовлетворяет условиям (14), то сумма $u(x, t)$ формального решения обладает следующими свойствами: вектор-функиия $u(x, t)$ непрерывно дифферениируема по $x$ u $t ; u_{x}^{\prime}(x, t)$ (соответственно, $u_{t}^{\prime}(x, t)$ ) абсолютно непрерывна по $x$ (соответственно, по $\left.t\right) ; u(x, t)$ удовлетворяет уравнению (1) почти всюду и условиям (2)-(4); т.е. и $(x, t)$ является классическим решением задачи (1)-(4), когда уравнение (1) удовлетворяется почти всюду.

\section{СПИСОК ЛИТЕРАТУРЫ}

1. Бурлуцкал $M$. ШI. Смешанная задача с инволюцией на графе из двух ребер с циклом// Докл. РАН. - 2012. - 447, № 5. - C. 479-482.

2. Бурлуцкал М. Ш. Метод Фурье в смешанной задаче для волнового уравнения на графе// Докл. РАН. - 2015. - 465, № 5. - С. 519-522.

3. Бурлуцкая М. Ш., Хромов А. П. Резольвентный подход в методе Фурье// Докл. РАН. - 2014. - 458, № 2. - C. $138-140$. 
4. Бурлуцкая М. Ш., Хромов А. П. Смешанная задача для волнового уравнения с суммируемым потенциалом в случае двухточечных граничных условий разных порядков// Диффер. уравн. - 2017. - 53, № 4. - С. 505-515.

5. Бурлуцкая М. Ш., Хромов А. П. Резольвентный подход для волнового уравнения// Ж. вычисл. мат. мат. физ. - 2015. - 55, № 2. - С. 51-63.

6. Головко Н. И., Голованева Ф. В., Зверева М. Б., Шабров С. А. О возможности применения метода Фурье к разнопорядковой математической модели// Вестн. Воронеж. гос. ун-та. Сер. Физ. Мат. 2017. - 1. - C. 91-98.

7. Кулаев Р. Ч. Теорема существования для параболической смешанной задачи на графе с краевыми условиями, содержащими производные по времени// Тр. Ин-та мат. мех. УрО РАН. — 2010. - 16, № 2. - С. 139--148.

8. Марченко B. А. Операторы Штурма-Лиувилля и их приложения. - Киев: Наукова Думка, 1977.

9. Найдюк Ф. О., Десятирикова E. Н., Проскурина Д. К. Численное решение задач о колебаниях// Вестн. Воронеж. гос. ун-та. Сер. Сист. анал. информ. техн. - 2013. - 1. - С. 55-60.

10. Наймарк М. А. Линейные дифференциальные операторы. - М.: Наука, 1969.

11. Покорный Ю. В. Дифференциальные уравнения на геометрических графах. - М.: Физматлит, 2004.

12. Провоторов В. В. Собственные функции задачи Штурма-Лиувилля на графе-звезде// Мат. сб. 2008. - 199, № 10. - С. 105-126.

13. Провоторов В. В., Волкова А. С. Начально-краевые задачи с распределенными параметрами на графе. - Воронеж: Научная книга, 2014.

14. Прядиев В. Л. Один подход к описанию конечной формы решения волнового уравнения на пространственной сети// Тр. Междунар. Крымской осенней школы-симпозиума (КРОМШ-15) «Спектральные и эволюционные задачи» (17-29 сентября 2004 г., Севастополь, Ласпи). - Симферополь, 2005. С. $132-139$.

15. Прядиев В. Л. Численная схема решения начально-краевой задачи для волнового уравнения на одномерной пространственной сети при обобщенно-гладких условиях трансмиссии // Вестн. Самар. гос. ун-та. Естественнонауч. сер. - 2008. - 67, № 8/2. - С. 195--202.

16. Прядиев В. Л., Коровина О. В. Структура решения смешанной задачи для волнового уравнения на компактном геометрическом графе в случае ненулевой начальной скорости// Изв. Саратов. ун-та. Нов. сер. Сер. Мат. Мех. Информ. - 2009. - 9, № 3. - С. 37-46.

17. Расулов М. Л. Метод контурного интеграла и его применение к исследованию задач для дифференциальных уравнений. - М.: Наука, 1964.

18. Хромов А. П. О сходимости формального решения по методу Фурье волнового уравнения с суммируемым потенциалом// Ж. вычисл. мат. мат. физ. - 2016. - 56, № 10. - С. 1795-1809.

19. Burlutskaya $M$. On a resolvent approach in a mixed problem for the wave equation on a graph// Mem. Differ. Equations Math. Phys. - 2017. - 72. - P. 37-44.

20. Zvereva M. String oscillations simulation with nonlinear conditions// Mem. Differ. Equations Math. Phys. - 2017. - 72. - P. 141-150.

Бурлуцкая Мария Шаукатовна

Воронежский государственный университет

E-mail: bmsh2001@mail.ru

Киселева Анна Викторовна

Воронежский государственный университет

E-mail: anyutka.kiseleva@gmail.com

Коржова Янина Павловна

Воронежский государственный университет

E-mail: ioanna1997@yandex.ru 